\title{
Accurate modeling of high frequency microelectromechanical systems (MEMS) switches in time- and frequency-domain
}

\author{
F. Coccetti ${ }^{1}$, W. Dressel ${ }^{1}$, P. Russer ${ }^{1}$, L. Pierantoni ${ }^{2}$, M. Farina ${ }^{2}$, and T. Rozzi ${ }^{2}$ \\ ${ }^{1}$ Technische Universität München, Lehrstuhl für Hochfrequenztechnik, Munich, Germany \\ ${ }^{2}$ Dipartimento di Elettronica ed Automatica, University of Ancona, Ancona, Italy
}

\begin{abstract}
In this contribution we present an accurate investigation of three different techniques for the modeling of complex planar circuits. The em analysis is performed by means of different electromagnetic full-wave solvers in the timedomain and in the frequency-domain. The first one is the Transmission Line Matrix (TLM) method. In the second one the TLM method is combined with the Integral Equation (IE) method. The latter is based on the Generalized Transverse Resonance Diffraction (GTRD). In order to test the methods we model different structures and compare the calculated Sparameters to measured results, with good agreement.
\end{abstract}

\section{Introduction}

The goal of our outgoing joint effort is the development and the application of efficient numerical tools for the analysis and modeling of complex open planar circuits, such as antennas, filters and more complex structure as Micro-ElectroMechanical-Systems (MEMS).These latter structures exhibit usually several geometrical details, finite dielectric layers, losses and thick metals and also strongly critical "aspectratios". Typically, it is very useful to deal with all these structures using different methods or solvers. The use of semianalytical methods like the integral equation method (IE) in connection with the method of moments (MoM) is usually restricted to strictly planar structures, Harrington (1982). However GTRD allows setting up an integral equation for truly 3-D structures, complementing the known advantages of MoM techniques (such as speed and reliability) with the flexibility of 3-D full-wave approach in the frequency domain. Its disadvantage lies in the need for some hypothesis on the structure, as it relies on knowledge of the Green Function describing the structure under test.

In (Farina and Rozzi, 2001) a 3-D GTRD formulation for boxed multilayer structures was presented that exploited the

Correspondence to: F. Coccetti
Green's function of a loaded box and was shown to be especially suited for MMIC and MEMS analysis. Space discretizing methods like the TLM method allow the numerical field modeling of structures with nearly arbitrary geometry (Johns, 1987; Russer, 2000). Their disadvantages appear when dealing with free space regions which increases considerably the 3-D-spatial domain of computation, thus increasing the number and the size of the elementary cells for the field modeling. The hybrid TLM-IE method combines the advantages of the TLM method in modeling nearly arbitrary complex structures and the advantages of the IE method in dealing with wide homogeneous regions, (Pierantoni et al., 1999). A minor drawback is the need of storing the timeevolution of the tangential field where TLM is coupled to the Green's function-based Integral Equation.

Three full-wave numerical tools were developed based to the aforementioned techniques:

(i) A solver based on the TLM method, which involves computer visualization (Mangold and Russer, 1999).

(ii) A solver based on the TLM-IE method (Pierantoni et al., 1999).

(iii) A general-purpose commercial program, including tools for pre and post processing, EM3-DS, distributed by MEM Research, based on GTRD method (Farina and Rozzi, 2001).

In order to compare accuracy and efficiency of the above three methods we have modeled a MEMS structure. In this contribution we discuss a MEMS capacitive switch, known to be challenging structure because of its severe aspect-ratio constrains (Sheen et al., 1990; Coccetti et al., 2000). Theoretical S-parameters are compared to experimental ones with very good agreement. 


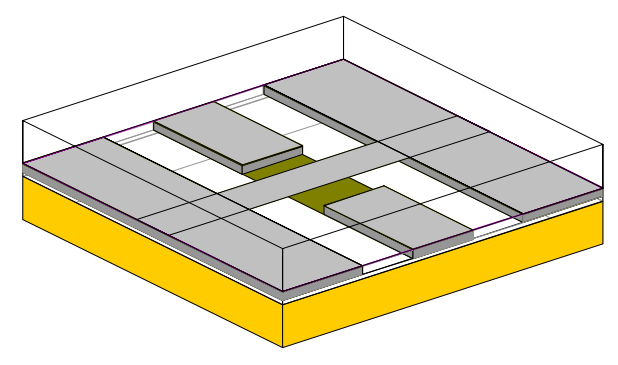

Switch “up” (On)

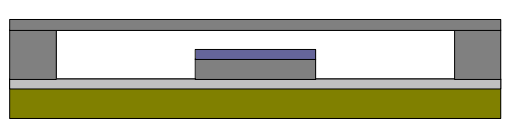

Switch "down” (off state)

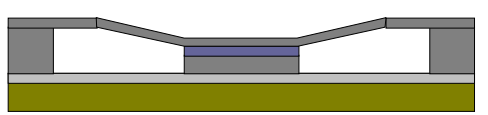

Fig. 1. MEMS switch and the cross section in the two working states.
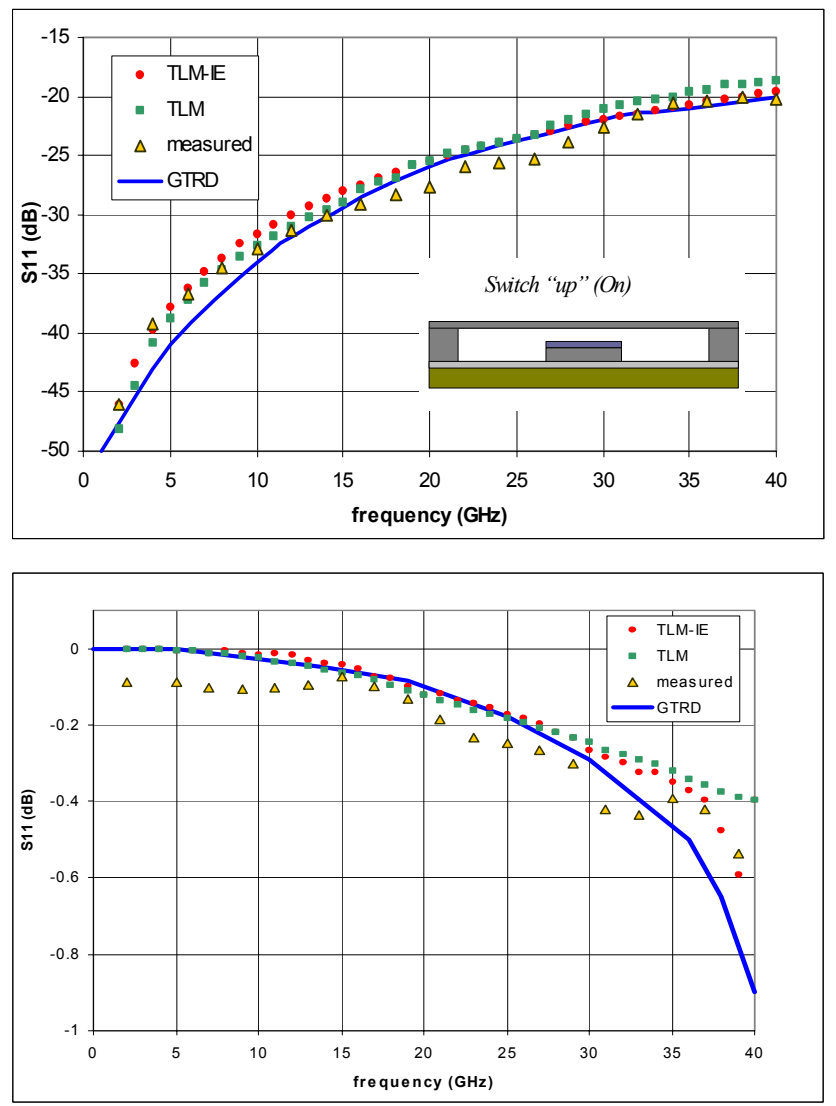

Fig. 2. On State: comparison of magnitude in $\mathrm{dB}$ for $\mathrm{S}_{11}$ (left) and $\mathrm{S}_{21}$ (right) vs. freq. $(\mathrm{GHz})$.

\section{Theory}

In the TLM method the evolution of the discretized electromagnetic field is modeled by wave pulses propagating on a mesh of transmission lines and scattered at the mesh nodes (Johns, 1987; Russer, 2000). In the TLM-IE method the 3$\mathrm{D}$ space is segmented into different sub-regions, where the best suited method, be it TLM or IE is applied. Inside the TLM-regions, the e.m. field is modeled by the TLM method. In IE-regions the e.m. field is analytically by means of the appropriate Green's function. The continuity of the field is
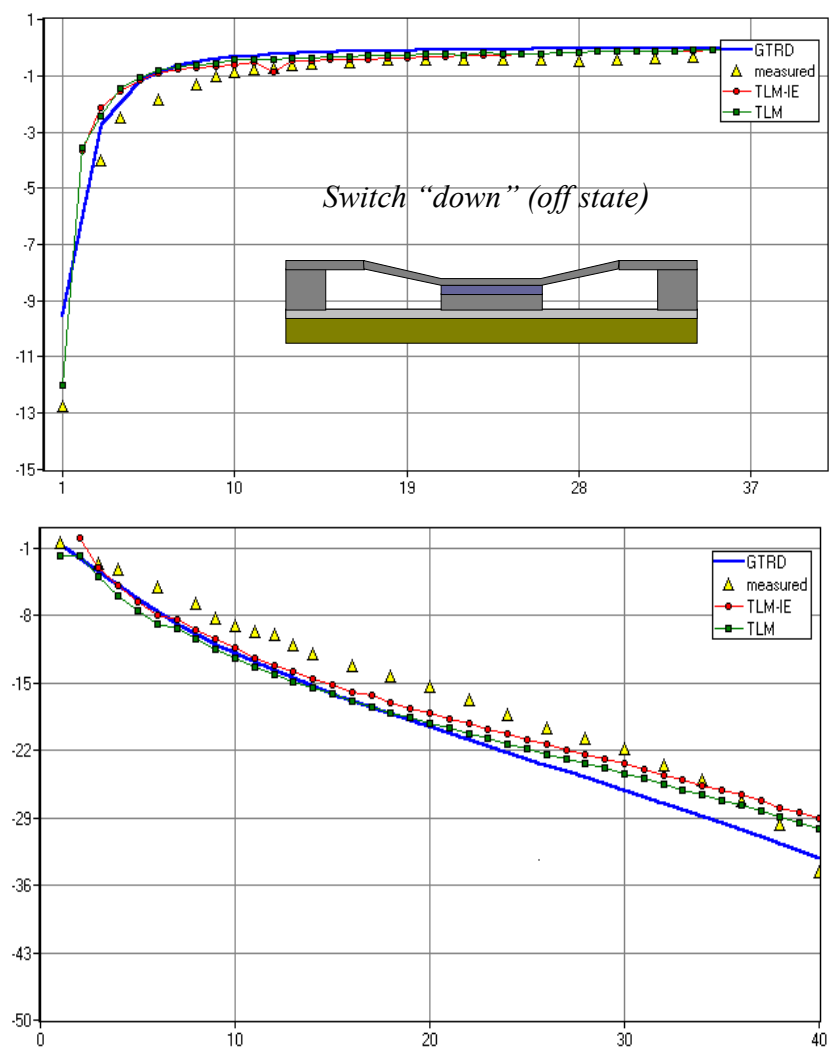

Fig. 3. Off State: comparison of magnitude in $\mathrm{dB}$ for $\mathrm{S}_{11}$ (left) and $\mathrm{S}_{21}$ (right) vs. freq. $(\mathrm{GHz})$.

applied at the interfaces between regions, providing appropriate integral equations for the tangential field.

The tangential field solution represents the exact boundary condition for the TLM algorithm (Pierantoni et al., 1999). In the GTRD approach the Green's function of a multilayer dielectric stack is calculated (Farina and Rozzi, 2001): the Green's function links fields within the stack to arbitrary current source distributions. Currents are defined in volumes describing lossy conductor regions, and by imposing Ohm's law to hold, an eigenvalue equation is obtained. The final step is to select appropriate excitation so as to transform the 

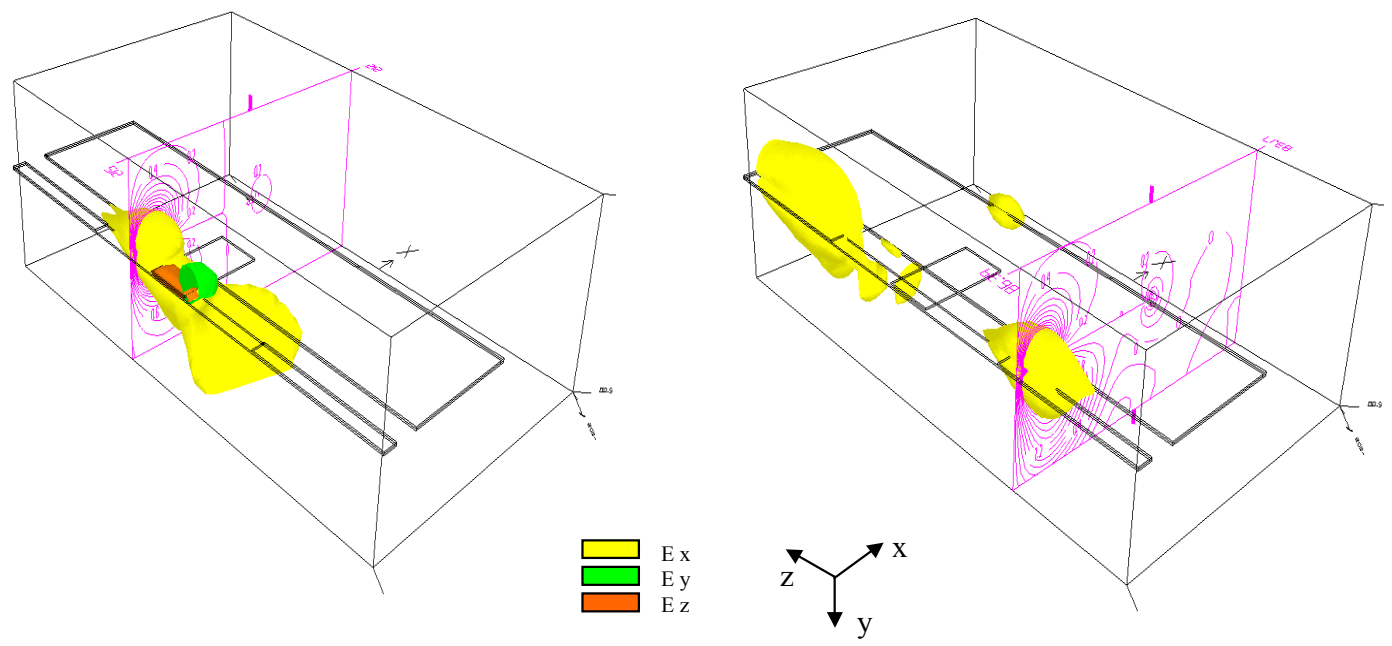

Fig. 4. On State 3-D field representation at two time instants: $t=t_{0}$ (left) and $t=t_{0}+2.5 \mathrm{ps}$ (right).
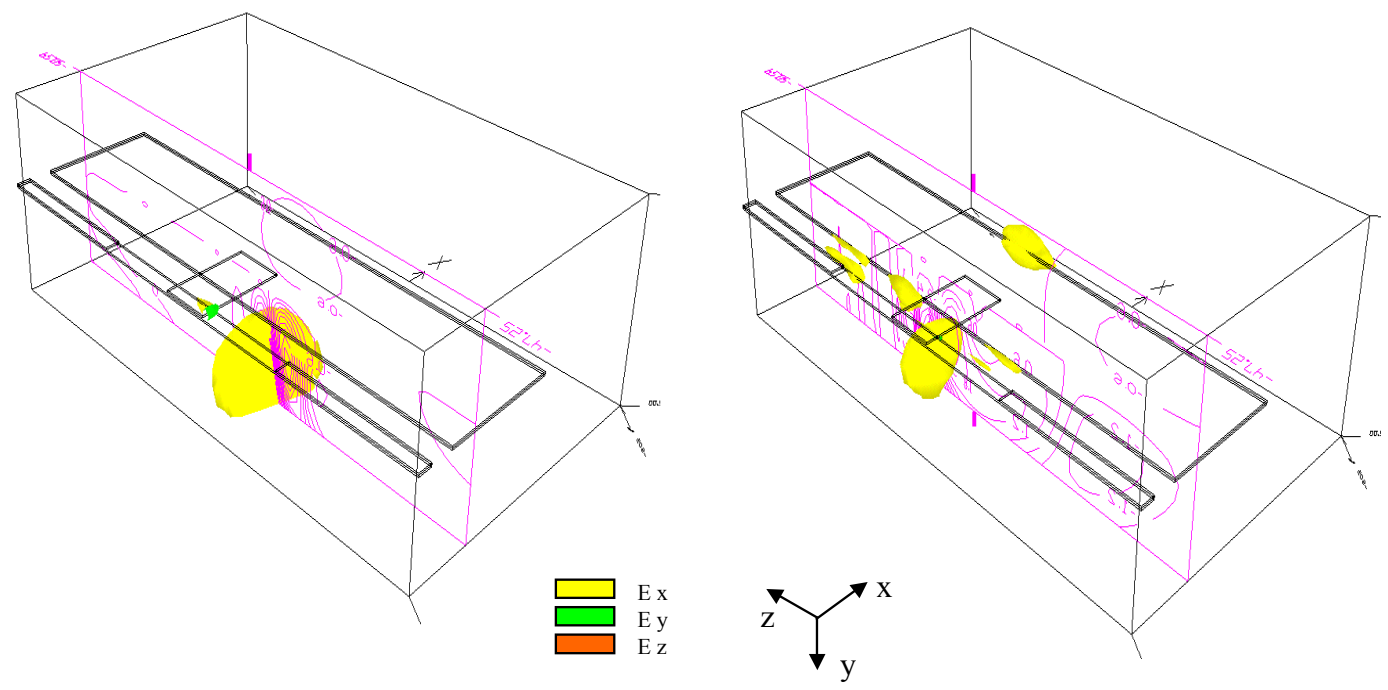

Fig. 5. Off State 3-D field representation at two time instants: $t=t_{0}$ (left) and $t=t_{0}+2.5 \mathrm{ps}$ (right).

eigenvalue equation into a deterministic one. In our case, excitations were selected to be standard delta-gap field sources, while the source discontinuity was removed by appropriate de-embedding.

\section{Results}

The three methods show high accuracy and agreement with experimental data. The TLM-IE and GTRD simulations have been performed by a $512 \mathrm{Mb}-\mathrm{RAM} 300 \mathrm{MHz}$ PC, while TLM over a HP-9000 C360.

It has to be mentioned that TLM and TLM-IE have their strongest point in the ability to model very complex structures, with nearly arbitrary shaped object in space. It should be remarked, however, that GTRD is a frequency-domain approach, so that the simulation time is dependent of the number of frequency points required, while TLM and TLM-
IE obtain the frequency-domain response as FFT of a timedomain evolution, implying advantages for broad band simulations.

Figure 1 shows the MEMS switch reported in Strohm et al. (1999). Figure 2 shows a comparison between TLM, TLMIE, GTRD and experimental data for the "on" state and the same comparison for the "off" state. In both cases GTRD computation required roughly $3 \mathrm{~h}$ CPU-time; the TLM-IE simulation requires about $2 \mathrm{~h}$ CPU-time. It is remarkable to observe that by using the TLM-IE method both the bulk Si-region and the free-space regions are modeled by means of the appropriate Green's function, thus drastically reducing the 3-D spatial domain of computation for the TLM algorithm.

A comparison of TLM method, TLM-IE method and GTRD method with experimental data shows a very good agreement in any analyzed structure. Slight differences on the accuracy are mostly due to the selection running param- 
eters (mesh size, number of time steps for TLM/TLM-IE, number of expansion functions for GTRD).

Any method has its own advantages and drawbacks. TLMIE, due to its hybrid nature, seems to offer a good trade-off between flexibility, accuracy and computation time.

Figures 4 and 5 show the time domain simulation of the electric field in the MEMS switch according to Fig. 4. The structure is excited with a Gaussian pulse $1.6 \mathrm{ps}$ of width. Due to the symmetry of the problem only half of the structure is depicted. The field distribution of the traveling pulse in the "on" state is depicted in Fig. 4. The shaded surfaces are the isopheres of $E_{x}, E_{y}$ and $E_{z}$. In the $x z$-plane and in the $y z$-plane respectively the isoclinal lines of $E_{x}$ are depicted.

The pulse is a snapshot of the time propagation in two time instant; $t_{0}$ just before the MEMS bridge, and at $t=t_{0}+2.5 \mathrm{ps}$ just after it. In a similar fashion the field distribution of the traveling pulse in the "off" state is depicted in Fig. 5.

Is pretty evident from these time domain plots, how the bridge in the two different state acts differently, producing a completely dual behavior, highlighted already in the given scattering parameters, with only $0.1 \mathrm{~dB}$ insertion loss and almost $20 \mathrm{~dB}$ isolation at $20 \mathrm{GHz}$.

\section{Conclusions}

The performances of the three full-wave approaches, TLM, TLM-IE and GTRD, have been compared for the case of planar and quasi-planar structures. Comparison with experimental results shows very good agreement. Besides the high accuracy a further advantage of TLM is its high flexibility with respect to general structures. A reduction of computation time by up to one order of magnitude with pure TLM can be achieved using system identification methods (Chtchekatourov et al., 2001).

\section{References}

Harrington, R. F.: Field Computation by Moment Methods, Krieger, 1982.

Farina, M. and Rozzi, T.: A 3-D integral equation-based approach to the analysis of real-life MMICs: application to microelectromechanical systems, IEEE Trans. Microwave Theory and Tech., December 2001.

Johns,P. B.: A symmetrical condensed node for the TLM method, IEEE Trans. Microwave Theory Tech., 35, 370-377, 1987.

Russer, P.: The transmission line matrix method, in: Applied Computational Electromagnetics, NATO ASI Series, pp. 243-269, Springer, Berlin, New York, 2000.

Mangold, T. and Russer, P.: Full-wave modeling and automatic equivalent-circuit generation of millimeter-wave planar and multilayer structures, IEEE Trans. Microwave Theory Tech., 47, 851-858, 1999.

Pierantoni, L., Lindenmeier, S., and Russer, P.: Efficient analysis and modeling of the radiation of microstrip lines and patch antennas by the TLM-integral equation (TLM-IE) method, Int. J. Numerical Modelling: Electronic Networks, Devices and Fields, 12, 4, 329-340, 1999.

Sheen, D. M., Ali, S. M., Abouzahra, M. D., and Kong, J. A.: Application of the three-dimensional finite-difference time-domain method to the analysis of planar microstrip circuits, IEEE Trans. Microwave Theory Tech., 38, 7, 849-857, 1990.

Coccetti, F., Vietzorreck, L., Chtchekatourov, V., and Russer, P.: A numerical study of MEMS capacitive switches using TLM, Proc. 16th Annual Review of Progress in Applied Computational Electromagnetics, Monterey 2000, 580-586, 2000.

Strohm, K., Rheinfelder, C., Schurr, A., et al.: SIMMWIC capacitive RF switches, 29th European Microwave Conference, Munich 1999, 2, 411-414, 1999.

Chtchekatourov, V., Coccetti, F., and Russer, P.: Direct Yparameters estimation of microwave structures using TLM simulationand Prony's method, Proc. 17th Annual Review of Progress in Applied Computational Electromagnetics, Monterey 2001, 461-467, 2001. 